\title{
DISCUSSÃO SOBRE O POTENCIAL DA METODOLOGIA BIM NA INSPECÇÃO E DIAGNÓSTICO DE EDIFÍCIOS
}

\author{
Ana Sousa $^{(1)}$, Eva Barreira ${ }^{(1)}$, Ricardo M. S. F. Almeida ${ }^{(1)}{ }^{(2)}$, João Poças Martins ${ }^{(1)}$ \\ (1) Faculdade de Engenharia da Universidade do Porto, Porto \\ (2) Departamento de Engenharia Civil, Instituto Politécnico de Viseu, Viseu
}

\begin{abstract}
Resumo
A inspeção e diagnóstico de edifícios é um instrumento fundamental, quer para a manutenção, quer para a preparação de uma intervenção de reabilitação. A crescente dinâmica associada à reabilitação urbana tem valorizado esta componente, justificando a procura de novas metodologias e técnicas de levantamento das anomalias. Para além da identificação dessas anomalias, a quantificação da severidade, também designada por grau de degradação, pode ser uma informação muito relevante para o projetista.

Pelas suas inúmeras potencialidades, a termografia de infravermelhos (TIV) tem motivado um interesse crescente como tecnologia fundamental na inspeção e diagnóstico dos edifícios. A sua utilização nesta área é, no entanto, sobretudo qualitativa, resultando apenas da observação direta das imagens térmicas. Contudo, a interpretação quantitativa dos resultados pode acrescentar informação valiosa, nomeadamente para estabelecer critérios de avaliação do grau de degradação dos elementos.

A metodologia BIM (Building Information Modelling) pode e deve ser aproveitada nesta área, nomeadamente através da inclusão no modelo digital do edifício da informação resultante da inspeção e diagnóstico. A incorporação das imagens térmicas no modelo é um exemplo claro da mais-valia associada a esta integração. Neste trabalho discute-se o potencial, as vantagens e as eventuais limitações, da metodologia BIM na inspeção e diagnóstico de edifícios e é apresentada uma proposta de integração desta informação no modelo BIM do edifício.
\end{abstract}

\section{Introdução}

O cadastro do património existente em Portugal encontra-se desatualizado ou, por vezes, até mesmo inexistente, devido a um ineficiente plano de gestão e manutenção do edificado. Por essa razão, torna-se evidente a necessidade de desenvolvimento dum sistema de gestão de informação para suporte das ações de manutenção e conservação.

Em Portugal o futuro da construção passa pela reabilitação do edificado existente. Isto implica um conhecimento técnico alargado acerca dos materiais e das metodologias de intervenção. A realização de uma intervenção deste caracter está dependente de uma adequada inspeção prévia 
do local, possivelmente complementada com ensaios, para sustentar um correto diagnóstico do real estado de degradação dos elementos. A inspeção dos edifícios permite detetar atempadamente anomalias, contribuindo para a definição de um plano de manutenção e auxiliando no planeamento das ações de prevenção, minimizando os custos de intervenção.

Durante a inspeção deve ser recolhida toda a informação que permita caracterizar as anomalias principais, a sua extensão e causas prováveis. De forma a realizar este estudo é necessária uma base onde toda a informação do edifício a reabilitar esteja atualizada e de acordo com o existente. Deste modo o presente artigo pretende evidenciar as potencialidades da metodologia BIM (Building Information Modelling) como ferramenta de gestão da informação que possa ser relevante num projeto de reabilitação um edifício. Essa agregação de informação num só modelo auxilia na gestão da obra e na manutenção do empreendimento.

A quantidade de informação processada e trocada durante o período de projeto pode atingir grandes quantidades e é trabalhada por diversos intervenientes. Esta diversidade de atores é sempre uma dificuldade inerente a qualquer projeto, e que aumenta com a dimensão do mesmo. A resolução deste problema pode passar por agregar toda a informação numa única plataforma, garantindo-se assim uma maior eficiência e fiabilidade no processo de gestão e produção.

Esta necessidade já há muito evidenciada pela indústria da Arquitetura, Engenharia e Construção encontrou a sua solução na metodologia BIM.

Esta tecnologia garante a minimização de tempo despendido e dos desperdícios, primando pela flexibilidade e capacidade de mudança, mas tal só é possível se todas as partes envolvidas estiverem cientes das potencialidades dos softwares e for assegurado um bom sistema organizacional devidamente enquadrado neste novo conceito. Torna-se essencial conseguir controlar a informação de um projeto numa única plataforma e a comunicação mais eficaz entre as diferentes especialidades, garantindo a fluidez na passagem da informação.

A dinamização desta tecnologia leva à expansão do BIM noutras áreas de intervenção como é o caso de inspeção e diagnóstico.

A reabilitação urbana exige cada vez mais uma avaliação pormenorizada do edificado existente para garantir maior fiabilidade na execução do projeto, minimizando por esta via eventuais contratempos em obra. Assim, é fundamental a recolha de informação quantitativa. No caso particular de uma reabilitação, a quantidade de informação existente é agravada pela introdução dos dados obtidos durante a fase de inspeção e diagnóstico do edifício existente.

A resolução deste problema passa então por adicionar esta informação aos restantes elementos do projeto, numa mesma plataforma, permitindo uma rápida e fácil identificação dos dados.

A marcação e identificação das anomalias levantadas, assim como a quantificação da sua severidade, pode ser uma informação muito relevante para o projetista, dando lhe indicação dos elementos em que a atuação é prioritária e qual o grau de intervenção necessário.

Neste artigo propõe-se a agregação da informação quantitativa e gráfica proveniente de inspeção através da TIV (Termografia de Infravermelhos) no modelo BIM de um edifício, com a finalidade de auxiliar quer para a manutenção, quer para a preparação de uma intervenção de reabilitação.

\section{Building Information Modelling (BIM)}

BIM designa todos os processos de produção e gestão da informação durante o ciclo de vida da construção. 
O BIM é uma filosofia de trabalho que pressupõe a interação entre os vários agentes existentes em todas as fazes da vida de um edifício. Representa uma abordagem à gestão da informação da construção que se estende a todo o ciclo de vida, pressupondo que em fase de projeto e construção, arquitetos, engenheiros e construtores trabalhem em conjunto na elaboração de um modelo virtual preciso, que origina uma base de dados contendo tanto informações geométricas, como informações necessárias para a realização de orçamentos, cálculo energético e gestão da construção em todas as suas fases [1].

As aplicações mais correntes utilizam o modelo tridimensional do edifício como um repositório dinâmico para integração da informação da construção e representação digital das características físicas e funcionais de uma edificação. Os processos de trabalho envolvendo ferramentas BIM desenvolvem-se à volta deste modelo que integra uma parte substancial da informação relevante.

Esta metodologia é colaborativa, inteligente e sustentável, mas também muito desafiante. Os modelos BIM mais ambiciosos dizem-se "nD". Esta designação caracteriza o âmbito dimensional que vai além das clássicas três dimensões do espaço euclidiano. A quarta dimensão de um modelo BIM é o fator tempo. A capacidade de retratar o ciclo de vida da construção, estratificando o modelo por fases de execução da construção, permite uma visão única da evolução do edifício ao longo do tempo. Por outro lado, esta arquitetura pode ser aproveitada no contexto das aplicações de planeamento dos processos produtivos. A quinta dimensão de um modelo BIM corresponde à associação de custos. A capacidade de atribuir valores aos elementos do edifício apoia e agiliza, em larga medida, os processos de orçamentação. Esta funcionalidade beneficia da extração automática de quantidades para evitar erros de medição e a propagação de inconformidades, assegurando estimativas sempre coerentes com o estado atual do projeto. A escala temporal e os custos são, atualmente, as dimensões "extra" mais divulgadas, no entanto, o potencial dos BIM permite outras dimensões, sobretudo a nível de simulações e cálculo, sendo possível utilizar a extração automática de quantidades e a atribuição de parâmetros aos elementos para proceder à simulação de cenários para análise estrutural e energética.

A abrangência do BIM permite abarcar um volume significativo da informação referente ao ciclo de vida do edifício num único modelo. A partilha deste modelo com os vários colaboradores permite que o trabalho seja realizado a partir da mesma plataforma, minimizando os erros e omissões provenientes da interpretação e tradução deficiente da informação, e permitindo uma otimização da harmonia do modelo à medida que novos dados são acrescentados.

\subsection{Potencial da Metodologia BIM}

As ferramentas BIM têm um enorme potencial e um leque vasto de funcionalidades que vai muito além das permitidas pelas tradicionais ferramentas $\mathrm{CAD} 3 \mathrm{D}$, beneficiando dos princípios de automação que regem os modelos BIM.

Entre as vantagens identificadas para a indústria da construção, associadas à adoção deste tipo de tecnologia, contam-se as seguintes [2], [3]:

- Pesquisa e obtenção eficientes de documentos específicos;

- Propagação de alterações rápida e direta;

- Automatização de fluxos de trabalho; 
- Compilação da informação relevante;

- Integração de processos de produção e de gestão documental que resultam numa economia de esforços ao nível administrativo;

- Simplificação da recolha de informação produzida em projetos anterior ou proveniente de fontes de informação externas;

- Criação de condições favoráveis para a realização simultânea do trabalho de diversos projetistas, resultando em prazos mais curtos para o desenvolvimento de projetos;

- Eliminação da introdução repetida de dados, evitando-se os erros associados;

- Redução de esforços redundantes relacionados com a repetição de tarefas de projeto e com as verificações das especificações elaboradas;

- Aumento de produtividade devido a uma partilha de informação mais rápida;

- Simplificação da introdução de modificações em projetos;

- Melhoria da cooperação interdisciplinar;

- Redução da duração da fase de construção;

- Maior facilidade na deteção de incompatibilidades, reduzindo os erros;

- Maior facilidade de compreensão do resultado final através da visualização do modelo;

- Benefícios para planeamento, gestão e manutenção de edifícios;

- Maior rapidez de partilha de informação, logo maior rapidez na correção de erros;

- Possibilidade de extração automática de mapas de quantidades do modelo, e atualização automática dos mesmos aquando de uma alteração de projeto;

- Possibilidade de simulação animada do faseamento construtivo do modelo;

- Possibilidade de organizar informação de forma que se entender mais pertinente;

- A possibilidade de cálculo de quantidades de materiais permite simular os custos de diversas soluções/modelos auxiliando no estudo de soluções mais económicas.

Embora a primeira entidade a sentir estas vantagens seja o projetista, elas fazem-se sentir também em fase de construção. A existência de uma base de dados partilhada pelos diversos intervenientes, contendo a generalidade das informações produzidas durante o processo construtivo, alterará radicalmente a forma como é feita atualmente a gestão de informação.

Embora o conceito BIM seja bastante inovador e potencialmente indutor de inúmeras vantagens para os seus utilizadores, existem, ainda, diversas limitações/desafios ao seu uso e implementação. Com a utilização destes softwares extingue-se o anterior conceito de registo de revisões de projeto, feito muitas vezes pelo envio do ficheiro atualizado. No caso do BIM o modelo de trabalho encontrar-se-á, à partida, sempre atualizado a cada alteração feita pelos diversos intervenientes, evitando a consulta/utilização de versões obsoletas/ultrapassadas.

Outra das dificuldades sentidas na sua utilização é a compatibilização e troca de dados entre os diversos softwares que obriga as equipas projetistas a trabalharem com o mesmo sistema de modo a possibilitar a compatibilização da informação e evitando perdas de informações. $\mathrm{O}$ mesmo acontece com a troca de dados entre um programa de cálculo e os programas de modelação, os dados poderão não ser transferidos/assumidos na íntegra na transição de softwares. Outro obstáculo a ser ultrapassado é a constituição de uma biblioteca de objetos standard com elementos adequados e adaptados à legislação Portuguesa, garantindo que as diferentes entidades acedem à mesma base de dados de objetos, uniformizando, assim, os projetos e minimizando potenciais perdas de informação na sua partilha. 


\subsection{BIM na Inspeção e Diagnóstico de Edifícios}

As ferramentas BIM estão pensadas para a aplicação a projeto de edifícios novos, pelo que a sua utilização na reconstrução de edifícios existentes se torna mais trabalhosa. A lógica de reconstrução passa por modelar objetos de natureza única, muitas vezes de contornos complexos e cuja parametrização é mais complexa do que a da construção corrente. No entanto, estas ferramentas têm um enorme potencial na gestão da informação geométrica e não geométrica do património arquitetónico.

À reabilitação está inerente em muitos casos a ausência de conhecimento sobre as condições atuais do edifício, que advêm principalmente da inadequada ou inexistente atualização da informação referente ao estado do edifício, agravada pelo inevitável envolvimento de vários profissionais ao longo da sua vida. Assim, estes obstáculos traduzem-se na ausência de documentação adequada para o apoio à equipa de projeto, que se depara com a tarefa de encontrar informação sobre o edifício que possa estar desatualizada ou até mesmo incompleta, em múltiplos formatos e vinda de múltiplos profissionais com intervenções prévias no edifício. Estas razões levam a que a aplicação do BIM na inspeção e diagnóstico de edifícios esteja dependente da construção do modelo do edifício.

Com o objetivo de superar estes obstáculos tem-se aplicado técnicas que permitem a recolha detalhada e não destrutiva da geometria do edifício, bem como a geração de representações tridimensionais das condições atuais do mesmo, possibilitando assim à equipa de projeto uma rápida análise do edifício. O laser scanning, a fotogrametria, a videogrametria e a triangulação ótica, entre outros, são exemplos destes métodos de levantamento. Estas são tecnologias que exigem equipamento frágil e dispendioso, bem como operadores formados e, em alguns casos, longos períodos de levantamento, pelo que a sua aplicação ainda não está vulgarizada. A partir destes métodos é possível a criação de nuvens de pontos a utilizar numa ferramenta BIM como fonte para a modelação.

Em [2] foi comparado o modelo criado a partir dos projetos e o modelo criado a partir do levantamento e verificou-se a importância de trabalhar sobre levantamentos, uma vez que as condições reais eram significativamente diferentes, por exemplo em termos de cotas de soleira. O modelo realizado a partir do levantamento demonstrava a existência de assentamentos de fundação.

O conceito Heritage Building Information Modeling (HBIM) surgiu no fim da década de 2000 e refere-se à aplicação de ferramentas e processos BIM ao património arquitetónico, como meio de apoiar a sua conservação, reabilitação e reutilização sustentáveis, preservando o seu valor cultural e estético, memória e significado. Esta abordagem procura a criação de uma base de dados para objetos patrimoniais que permite: validar o modelo geométrico associando-lhe fontes de informação de diversa natureza; ensaiar hipóteses quando as fontes não são conclusivas e, posteriormente, adicionar nova informação; concentrar a informação relativa ao processo de modelação e suas opções; e facilitar o acesso e cruzamento dos dados [4].

A documentação e registo referem-se às fontes de informação de suporte do modelo e podem ser de naturezas diversas: entrevistas a investigadores, documentação escrita; ilustrações, levantamentos tradicionais, fotografias, desenhos técnicos, modelos fotogramétricos, levantamentos por laser scanning. Fontes de diversas naturezas podem ser utilizadas de forma complementar. É importante que a correlação entre a geometria do modelo e a fonte de origem seja clara, permitindo a sua reanálise à posteriori.

A aplicação da metodologia BIM na inspeção e diagnóstico de edifícios começou já a dar os seus primeiros passos. Segundo [5] já existem empresas em Portugal a implementar a 
metodologia nesta área de negócio, seguindo um processo de trabalho semelhante ao exposto na parte seguinte deste artigo, contudo não são ainda conhecidos os resultados da sua aplicação. Através da revisão da literatura realizada, verificou-se que a nível mundial, a TIV tem sido usada na deteção de patologias que posteriormente são adicionadas ao modelo criado sobre a nuvem de pontos. Contudo, existem também estudos nos quais esta nuvem de pontos é produzida a partir de imagens termográficas, ou fundida com nuvens de pontos de um edifício para produzir um modelo 3D termográfico, também conhecido como ortotermograma [6]. Esta ortotermografia representa um mapa 3D da fachada ou de qualquer estrutura do edifício, juntamente com sua textura termográfica que permitem que defeitos sejam detetados e analisados de forma eficaz. O ortotermograma pode ser mapeado através de laser scanning ou fotogrametria, contudo neste âmbito, o método genericamente utilizado para a criação de nuvens de pontos é o laser scanning, devido a sua maior precisão e autonomia.

Lagüela et al. [7,8] desenvolveram uma nova estrutura para mapear automaticamente imagens térmicas infravermelhas nas nuvens de pontos de um edifício. Outra estrutura semelhante foi destacada por Aguilera et al. [9] que fundiram nuvens de pontos do laser scanning com imagens de infravermelho para produzir modelos 3D termográficos para estudar o desempenho energético dos edifícios

\section{Metodologia Proposta}

A reabilitação exige cada vez mais uma avaliação exaustiva do edificado existente para garantir maior fiabilidade na execução do projeto, minimizando as "surpresas" em obra. Assim, é de grande relevância a recolha quantitativa de informação e sobretudo a qualidade da mesma. Em termos de conteúdo, essa qualidade irá sempre depender dos equipamentos utilizados, do operador, da quantidade de amostragem recolhida, entre outros fatores. Contudo, é na forma de transmissão da informação que poderá estar o elemento diferenciador. Nesse sentido olhou-se para a tecnologia BIM como esse elemento, pois permite agregar numa única plataforma toda a informação não gráfica, conseguindo ainda aliar uma comunicação geométrica e gráfica mais poderosa e detalhada.

A metodologia proposta neste artigo tem como objetivo facilitar a identificação das anomalias em formato tridimensional, bem como permitir observar o registo fotográfico das mesmas a partir do software REVIT (Figura 1).

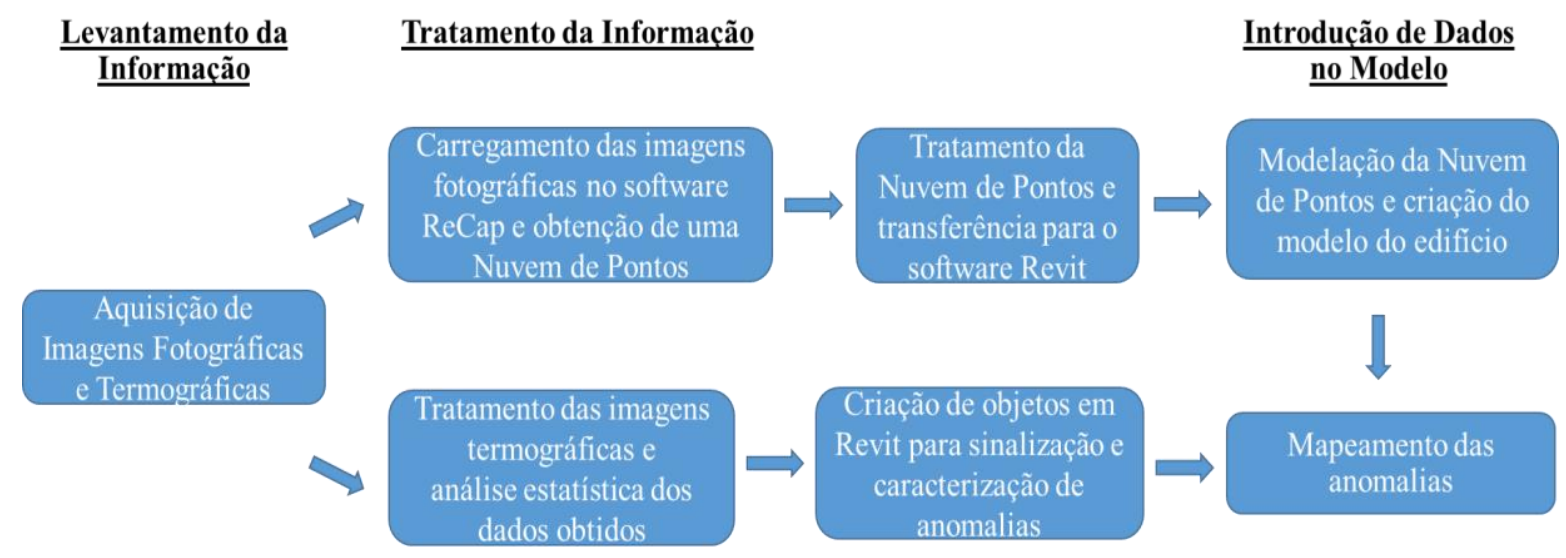

Figura 1: Metodologia proposta. 


\subsection{Levantamento da Informação}

Para a realização do modelo BIM de um edifício é necessário primeiramente a realização do trabalho de campo, no qual se propõe que, para além da aquisição de todas as informações e documentação disponível, se aplique a técnica de fotogrametria, obtendo o maior número possível de fotografias de todos os ângulos do compartimento/s que se pretende representar, sendo importante garantir a numeração correta das fotografias.

Na investigação que se pretende levar a cabo, não se exige muita definição e exatidão no modelo obtido, mas sim que este seja célere e automatizado. Desta forma, optou-se por propor uma tecnologia menos dispendiosa do que o laser scanning e de aplicação mais simples. A técnica que se propõe aplicar tem como desvantagem não permitir obter um modelo com o nível de detalhe só possível com o varrimento laser e a necessidade de se usar objetos de forma a obter pontos de referência e contrastes na nuvem obtida.

Para além da informação necessária para a criação do modelo é também nesta fase que dever ser realizada a identificação visual das anomalias e das suas características. A metodologia proposta pressupõe a utilização da TIV na inspeção das patologias, pelo que nesta fase deveram ser adquiridas as imagens termográficas dos diversos elementos.

\subsection{Tratamento da Informação}

Nesta etapa do processo pretende-se a construção do modelo a partir da nuvem de pontos. Para tal propõe-se a obtenção da nuvem de pontos através do software ReCap da Autodesk. A obtenção da nuvem de pontos é um processo automático, contudo manipular e tratar todos os dados obtidos dos diferentes levantamentos, com o objetivo de obter um único modelo consistente, não é um processo automatizado, sendo necessário recorrer a aplicações que

permitem a semi-automatização desta tarefa. É possível obter um modelo virtual representativo do real mas é uma tarefa que exige algum tempo e conhecimento do utilizador.

A par da construção do modelo virtual é também nesta fase que se irá realizar o tratamento dos dados provenientes de inspeção.

As imagens obtidas da TIV assim como os valores de temperatura daí retirados serão interpretados através de um tratamento estatístico (por exemplo a análise de Clusters), que permitirá organizar as patologias encontradas em famílias e definir uma escala de gravidade/deterioração dessas mesmas patologias.

\subsection{Introdução de dados no BIM}

Após a obtenção de um modelo BIM representativo da construção real pode dar-se início ao mapeamento das anomalias com a colocação do objeto identificador e upload do registo fotográfico devidamente numerado e caracterização completa da anomalia.

A introdução manual das anomalias nesse modelo será um processo análogo ao efetuado em ficheiros bidimensionais, mas agora em formato tridimensional e com maior facilidade de localização geométrica ou espacial da anomalia.

Pretende-se para tal criar identificadores de anomalias (objetos para mapeamento de anomalias) no modelo de REVIT. A esses identificadores serão atribuídos diversas cores de acordo com a classificação obtida pela anomalia segundo os graus de degradação atribuídos pelos parâmetros de avaliação internamente definidos.

O mapeamento das anomalias pode ser feito de forma manual colocando-se os objetos no modelo, ou pela construção automática da matriz de informação no Excel através de macros. Disto depende a exportação do formato da tabela de identificadores REVIT para Excel e o seu 
preenchimento e posterior importação da tabela de Excel devidamente caracterizada para o REVIT.

Considerou-se a colocação das características geométricas obtidas das patologias, porém, a complexidade que a representação explícita da geometria representa e o tempo inerente na modelação iria complicar o processo. Para observação das características visuais da patologia podem ser consultadas as fotografias específicas já indexadas ao modelo.

\subsection{Limitações da metodologia em estudo}

As limitações da utilização da metodologia proposta prendem-se com a necessidade de ser realizada por operadores qualificados, tanto no que se refere às técnicas de levantamento, como no posterior tratamento da informação recolhida, não sendo possível a total automatização das várias fases da metodologia.

Os modelos obtidos apresentam uma grande quantidade de informação ao nível da nuvem de pontos e no modelo digital BIM do projeto de reabilitação, sendo necessário equipamento informático com grande capacidade de suporte para partilha, análise e manipulação destes modelos.

Uma das grandes dificuldades desta técnica é também a manipulação da nuvem de pontos, necessária para a criação do modelo. Para além disto são necessárias muitas fotografias para a aquisição de uma geometria e esta, após tratamento não é precisa devido aos vários erros na modelação "em cima da nuvem". Numa fase de estudo inicial a precisão não é requerida, mas numa fase avançada do projeto de reabilitação seria necessária.

\section{Conclusões}

O BIM é uma metodologia capaz de dar resposta a várias necessidades do setor da construção, particularmente no que se refere à conservação e manutenção do património, sendo de grande utilidade na documentação e gestão de edifícios.

A funcionalidade e eficácia deste sistema depende da quantidade e da qualidade da informação que lhe é fornecida, nomeadamente do nível de detalhe dos modelos associados. O custo e tempo inicial que é necessário investir no seu desenvolvimento, serão compensados a longo prazo, já que ao fornecer dados rigorosos para uma adequada reabilitação/manutenção do edificado permite a respetiva diminuição de custos.

É importante concluir que o levantamento de edifícios é muito importante nas intervenções de reabilitação, possibilitando a verificação do estado do edifício.

Através de um levantamento é possível determinar quais as intervenções relevantes e quais de revelam desnecessárias, facilitando o processo de visualização sem deslocação ao local.

Como evidenciado neste artigo, a utilização da metodologia BIM não é apenas vantajosa em projetos novos, podendo, quando combinada com outras técnicas, tais como a fotogrametria, criar modelos virtuais tridimensionais do edificado existente, coletando informações sobre a composição, auxiliando no estudo de viabilidade, reparação ou alteração para adequabilidade a novo uso ou função.

A comunidade técnica e científica já se encontra sensibilizada para os benefícios da aplicação das ferramentas BIM, existindo já várias empresas no país a trabalhar com esta metodologia. Contudo, as resistências inerentes a todas as mudanças levam a que a sua aceitação e implementação ainda se encontre numa fase incremental, principalmente por esta ser uma 
metodologia que exige formação especializada às equipas de trabalho e uma alteração relevante da organização e gestão.

\section{Referências}

[1] F. Dinis, J.P. Martins, B. Rangel e A. S.Guimarães, " Modelo Conceptual Para A Interação Com Informação De Projeto - Natural Bim Interface " in Livro de Atas ptBIM $2^{\circ}$ Congresso Portugues de Building Information Modelling (2018)

[2] J. Lopes, M.J.F. Silva, F.F.S. Pinho e P. Couto, " Utilização Da Metodologia Bim No Apoio À Reabilitação Funcional De Um Edifício" in Livro de Atas ptBIM $2^{\circ}$ Congresso Portugues de Building Information Modelling (2018)

[3] P. Monteiro e S. Oliveira, "Implementação Bim Na Tecnoplano - Desde A Ideia Até Ao Projeto Piloto" in Livro de Atas ptBIM $2^{\circ}$ Congresso Portugues de Building Information Modelling (2018)

[4] I. Rita, J. Rocha, A. Francisco e A. Tomé "Processos Digitais Hbim-Fff Para A Representação Física Do Património | O Caso De Estudo Do Palácio Nacional De Sintra" in Livro de Atas ptBIM $2^{\circ}$ Congresso Portugues de Building Information Modelling (2018)

[5] A. Silva e C.Moreira "Bim Como Supply Chain Manager De Informação" in Livro de Atas ptBIM $2^{\circ}$ Congresso Portugues de Building Information Modelling (2018)

[6] M. Hasan Shariq and Ben Richard Hughes, " Revolutionising building inspection techniques to meet large-scale energy demands: a review of the state-of-the-art" (2020)

[7] J S. Lagüela, L. Díaz-Vilariño, J. Armesto and P. Arias, " Thermographic 3D models as the foundation for Building Information Models" in 11th International Conference on Quantitative InfraRed Thermography (2012)

[8] S.LagüelaL, Díaz-Vilariño, J.Martínez and J.Armesto, " Automatic thermographic and rgb texture of as-built bim for energy rehabilitation purposes" (2013)

[9] D.González-Aguilera, S.Lagüela, P.Rodríguez-Gonzálvez and D.Hernández-López, " Image-based thermographic modeling for assessing energy efficiency of buildings façades" (2013) 